\title{
Dual Layer Microstrip Refflectarray Composed of Two Stacked Arrays with Minkowski and Square Shaped Radiating Element
}

\author{
A. Wahid ${ }^{a}$, M. K. A. Rahim ${ }^{a}$, F. Zubir ${ }^{a}$, M. F. Ismail ${ }^{a} *$ \\ ${ }^{a}$ Faculty of Electrical Engineering, Universiti Teknologi Malaysia, 81310 UTM Johor Bahru \\ *Corresponding author: ismail86mf@yahoo.com
}

Article history

Received: 8 March 2012

Received in revised form: 10 April

2012

Accepted: 18 July 2012

Graphical abstract

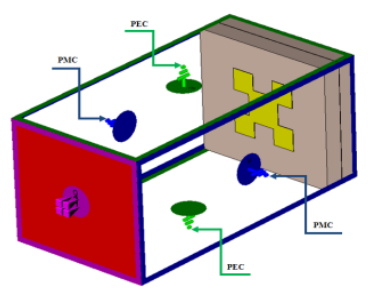

\section{Abstract}

A dual layer microstrip reflectarray composed of two stacked arrays with Minkowski and square patches of variable dimension is presented. The reflection phase coefficients on the reflector surface is achieved by tuning the dimensions of the patches. This technique is to broaden the bandwidth and to extend the reflection phase range compare to a conventional single layer reflectarray. From the simulation results of a unit cell composed of two stacked arrays of Minkowski and square patch showed that, $415^{\circ}$ reflection phase range is achieved and lower insertion loss which is lower than $0.9 \mathrm{~dB}$. Base on the simulated reflection phase coefficient, a dual layer microstrip reflectarray antenna with Minkowski and square radiating shape elements have been design and model using commercially available computer models of CST Microwave Studio. The reflectarray has been constructed using Taconic RF-35 substrate. From the radiation pattern at $11 \mathrm{GHz}$ frequency, it shows that the HPBW of $4.7^{\circ}$ in both plane, a side lobe level (SLL) of $-17 \mathrm{~dB}$ and a maximum directivity of $26.1 \mathrm{dBi}$.

Keywords: Reflectarray antenna; microstrip; Minkowski shape; square shape and dual layer

(C) 2012 Penerbit UTM Press. All rights reserved.

\subsection{INTRODUCTION}

The microstrip reflectarray antenna is one of the best candidates to replace the parabolic dish antenna. The antenna does not only found in the satellite communication applications but also can be use for point to point wireless communication and other communication areas. This type of antenna has been intensively studied by many antenna researchers in the academic and industrial sector.

Generally, the reflectarray consists an array of microstrip radiating element place on a planar dielectric sheet and illuminated by a feed antenna. They combine the best features of reflectors and phase array antenna. The reflectarray are less loss and less complex comparing to a phase array antenna because no feeding networks are necessary to connect the element on the surface and also it is lot more easily to manufacture than the reflector. Reflectarray are low-weight, low profile and less bulky which make them a suitable antenna candidate in certain application where a highly directive antennas are required.

In order to design the reflectarray, it is important to provide the reflection phase of the reflected wave. The simplest way to obtained the reflection phase is by varying the resonant length of the microstrip element [1-2]. This method had been used widely in many researchers because it is easier to implement and allowed simple manufacture by photo etching technique. Another famous method is by introducing variable stub length attached to the radiating microstrip element [3-4]. The problem with stubs is that it is bent to be accommodated in the array and they produce spurious radiation that degrades the crosspolarization levels. The tuning stubs also contribute to dissipative losses. Other alternatives have been proposed to control the phase distribution on a reflectarray, using phase shifters [5], or diodes as variable capacitors [6]. Some active reflectarray includes amplifiers, circulators, and phase shifters which can cause complication in the fabrication stage.

To design a practical microstrip reflectarray antenna, it is required to have a smooth $360^{\circ}$ phase range. This requirement is to prevent from the complication in the fabrication stage. Smooth phase range can decrease the fabrication tolerance and $360^{\circ}$ phase is avoid unoccupied unit cell on the reflectarray surface as stated in [7]. To improve the inconveniences of the nonlinear phase variations, a smoother phase variation within a range larger than $360^{\circ}$ can be obtained by stacking two or more arrays, as it will be shown in this paper. Many papers have been using this method [8], most of those papers used square or rectangular shape patch as a radiating element. In [7], a single layer reflectarray antenna with Minkowski shape radiating element is introduce which shows enhancement in the phase range comparing to a square shape radiating element. In this paper, a dual layer printed reflectarray based on Minkowski shape and square patches of variable size, as shown in Figure 1, is proposed in order to increase the bandwidth and to reduce the 
sensitivity to manufacture tolerances as a continuity of the work in [7]. The new dual layer printed Minkowski and square reflectarray elements are designed within X-band frequency range from 8.4 to $12.6 \mathrm{GHz}$ in CST Microwave Studio. It has been specified to operate at frequency of $11 \mathrm{GHz}$.

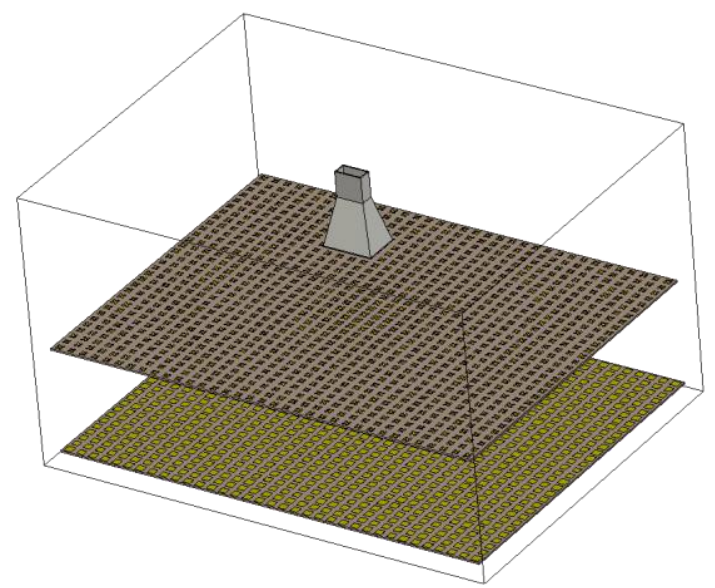

(a)

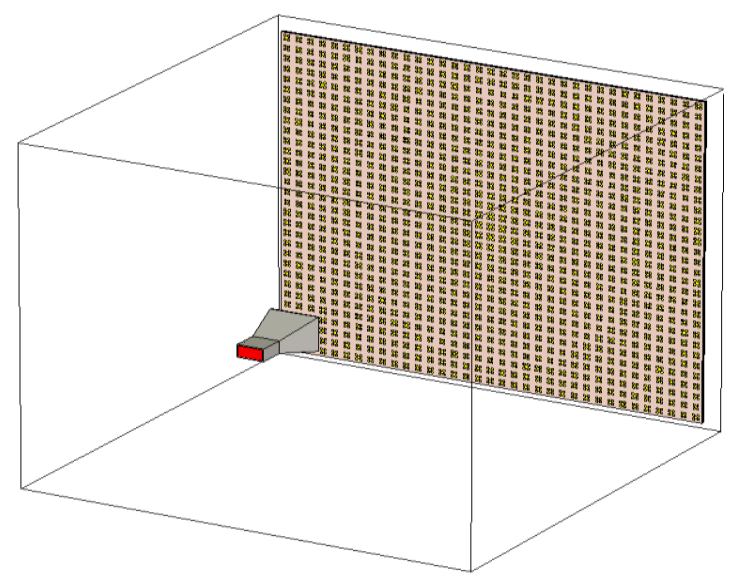

(b)

Figure 1 Dual layer printed reflectarray using patches of variable size. (a) Multilayer structure. (b) Reflectarray illuminated by a feed

\subsection{DUAL LAYER PRINTED UNIT CELL DESIGN} SPECIFICATION

As can be seen in Figure 2, a Minkowski shape is introduced as a radiating element for a dual layer unit cell configuration. This dual layer unit cell is called two staked arrays of Minkowski and square patches. In general, in Figure 2(a) the Minkowski patch is etched on the upper layer while the square patch is etched on the lower layer of the dielectric sheet. The element (Minkowski shape) dimension on the upper layer smaller 0.8 times of the element (Square shape) dimension on the lower layer which is near to the ground plane $\left(a_{1}=0.8 a_{2}\right)$. For the ease of comparison between the measurement and simulation results, the dimension of the unit cell configuration (dimension $11.43 \mathrm{~mm} \times 10.16 \mathrm{~mm}$ ) is half the dimension of the waveguide (dimension $22.86 \mathrm{~mm} x$ $10.16 \mathrm{~mm}$ ) which will be used in the measurement setup. Each Minkowski element is designed to be printed on a dielectric substrate of Taconic RF-35 with the thickness $(t=1.524 \mathrm{~mm})$, tangential loss $(\tan \delta=0.0018)$, and relative permittivity $\left(\varepsilon_{r}=\right.$ 3.54). In addition, the reflection coefficient of this unit cell configuration will be compared to a two staked arrays of two square patches. The configuration of this conventional configuration is shown in Figure 2(b). This is to distinguish the effectiveness of Minkowski radiating element shape for a dual layer unit cell in improving the reflection coefficient magnitude and phase.

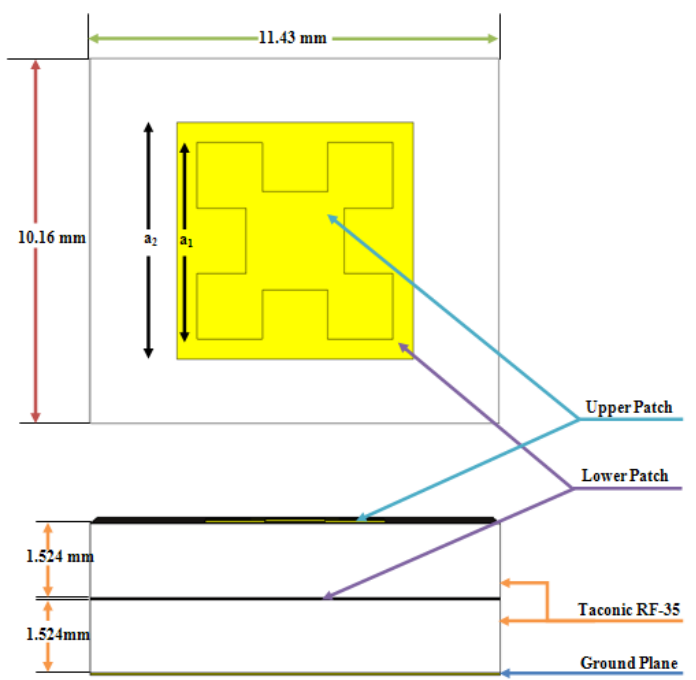

(a)

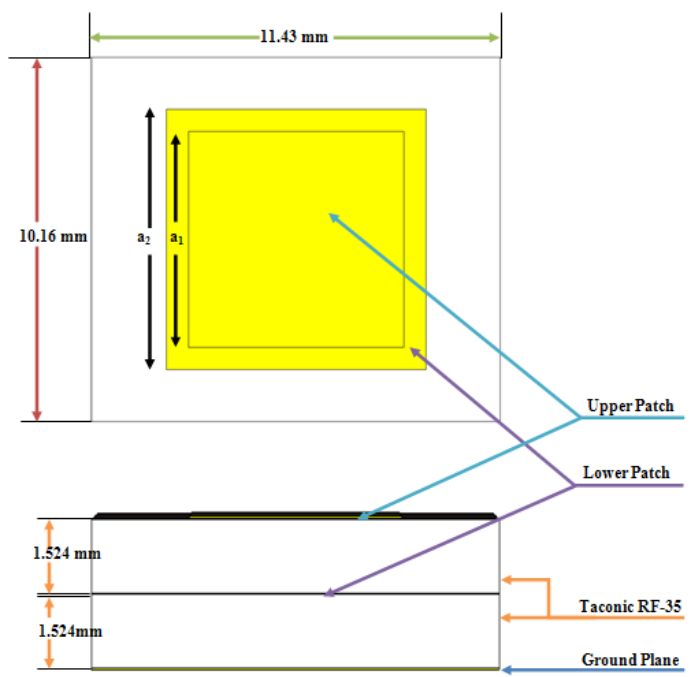

(b)

Figure 2 Dual layer printed unit cell configuration. (a) Two staked arrays composed of Minkowski and square patch. (b) Two staked arrays composed of two square patches

\subsection{DUAL LAYER PRINTED UNIT CELL} SIMULATION SETUP

In this work, the phase of the total field reflected from an infinite array with identical elements is computed using CST Microwave Studio. A dual layer unit cell is the building block of an infinite array with identical elements, consisting of the element itself (the two staked arrays configuration) and the part of the substrate and ground plane assigned to it, as determined by the grid dimension. To simulate the configuration, the dual layer unit cell needs to be modeled in the simulation environment. Next, suitable boundary conditions are chosen: 
two of the cell boundaries are perfect electric conductors (PEC), the other two are perfect magnetic conductors (PMC), as shown in Figure 3. This technique is called Infinite Array approach. The port is set at a convenient distance from the cell (about $1.5 \lambda$ to $2 \lambda$ ) to allow some propagating space, without making the computation domain unnecessarily large. An incident wave polarized along $\mathrm{z}$-axis is launched into this appropriately bounded waveguide. According to image theory, this arrangement is equivalent to an infinite array with identical elements. Moreover, the boundary conditions allow the propagation of a TEM mode. Thus, this setting is to make sure a unit cell will receive incidence energy from an illuminating feed just like a plane wave. The reflection coefficient is recorded at the surface of the cell, simply by setting the reference plane of the port accordingly.

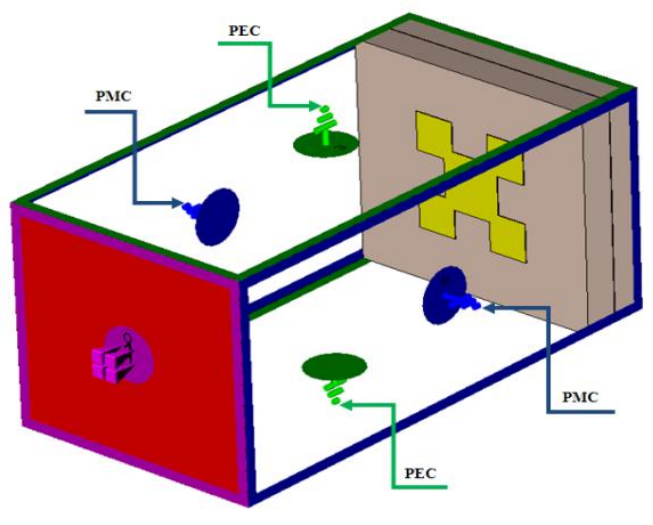

Figure 3 Boundary condition

The mesh cell of the design is determined prior for the before running the simulation of dual layer unit cell. Generally the default setting from the software is used in order to determine the mesh cell size of the modeled configuration. Since this parameter can give a major impact on the accuracy and speed of the simulation, the dual layer unit cell meshing size is determined by an adaptive mesh refinement in order to meet the simulation accuracy. This is important so that any further increment in mesh cell will not change the reflection phase response. Any factor or parameter which can lead to fabrication tolerance or failure in fabricating the whole structure of the reflectarray should be taken seriously. Therefore, an adaptive mesh refinement should be taken into consideration before determine the reflection phase of the simulation unit cell model of a reflectarray. In the simulation, the magnitude and phase of the reflection coefficient is obtained by varying the Minkowski patch dimension from $n=-2$ until $n=2$ where $n$ is the patch variation percentage. For example, $n=2$ means the patch itself has a size of $20 \%$ larger than the resonant size which is $n=0$ and vice versa.

\subsection{REFLECTION COEFFICIENT RESULT AND DISCUSSION}

Phase and magnitude of the reflection coefficient for two purpose configurations dual layer printed unit cell with stacked patches etched on Taconic RF-35 dielectric sheet are plotted and compared in Figure 4. The desired operating frequency of the reflectarray is chosen at $11 \mathrm{GHz}$. As a compromise between bandwidth and phase ranges a Taconic RF-35 substrate with a thickness of $h=1.524 \mathrm{~mm}$ has been chosen. The phase variation over patch variation is depicted in Figure 4(a). As can be seen in the figure, the maximal phase range is achieved at $11 \mathrm{GHz}$, which is the actual operating frequency for both configurations. The reflection losses for two staked arrays composed of minkowski and square patch is $-0.83 \mathrm{~dB}$ while for two staked arrays composed of two square patches is $-0.29 \mathrm{~dB}$. Both configurations shows a good reflection which is more than $50 \%$ of the signal will be reflected when strike the reflectarray. It can be observed in Figure 4(b), at $11 \mathrm{GHz}$ for two staked arrays composed of two square patches the phase is over only $330^{\circ}$. As for two staked arrays composed of Minkowski and square patch the total phase range is more than $360^{\circ}$. The phase range can be thus enhanced by around $85^{\circ}$, when introducing a Minkowski radiating element shape in the dual layer printed unit cell configuration.

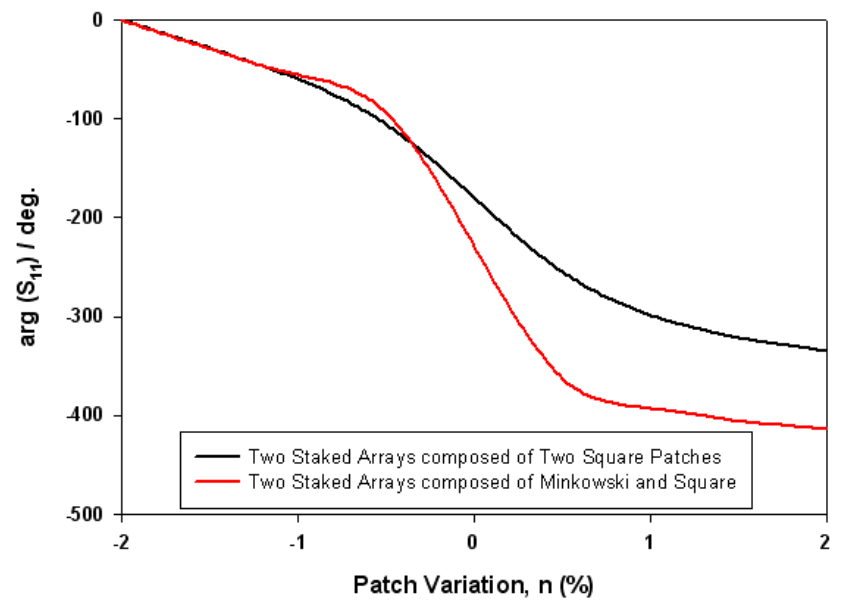

(a)

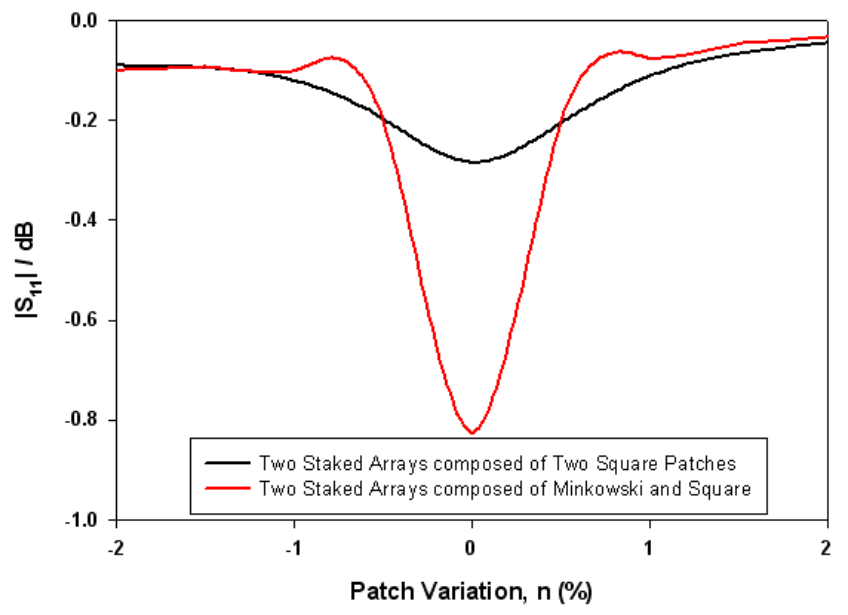

(b)

Figure 4 Simulation result for both purpose configurations. (a) Phase of the reflection coefficient. (b) Magnitude of the reflection coefficient

As shown in Table 1, at $11 \mathrm{GHz}$ the phase range for two stack arrays composed of Minkowski and square patch is $415^{\circ}$ range, which is more to the aim of $360^{\circ}$, the desired value that would allow realization of a large reflectarray without significant loss of performance. Since it has been shown dual layer printed unit cell with minkowski and square are more promising configuration for a practical reflectarray design, a dual layer 
printed reflectarray has been realize and modeled at $11 \mathrm{GHz}$ in the simulation environment. In addition, from the reflection phase characteristic for both configuration unit cell having Minkowski and square patch is steeper and not smooth to be compared to the configuration with square element. Hence if fabrication is needed to be done, the construction of the full reflectarray will face high fabrication tolerance which can lead to degradation of the reflectarray performance. In this paper, the reflectarray fabrication and measurement will not be discussed.

Table 2 Summary of the simulation reflection coefficient results

\begin{tabular}{|c|c|c|}
\hline $\begin{array}{c}\text { Dual Layer } \\
\text { Configuration }\end{array}$ & $\begin{array}{c}\text { Reflection } \\
\text { Phase (deg) }\end{array}$ & $\begin{array}{c}\text { Reflection } \\
\text { Magnitude (dB) }\end{array}$ \\
\hline Square-square & 330 & 0.29 \\
\hline Minkowski-square & 415 & 0.83 \\
\hline
\end{tabular}

\subsection{DUAL LAYER PRINTED REFLECTARRAY ANTENNA WITH MINKOWSKI AND SQUARE PATCH}

Having the characteristic as a parabolic reflector, a variety of antenna configurations can be used to feed a reflectarray: center feed, Cassegrain-feed, offset feed, to name just a few. The feeding with an offset horn antenna is one of the most widespread feeding configurations but in this work a center feed horn antenna is chosen. Its main advantage is that it will ease the installment of the horn in the simulation and measurement setup making it less complicated. In Figure 5 shows the existing pyramidal horn modeled in the simulation environment and the antenna specification is shown in Table 2.
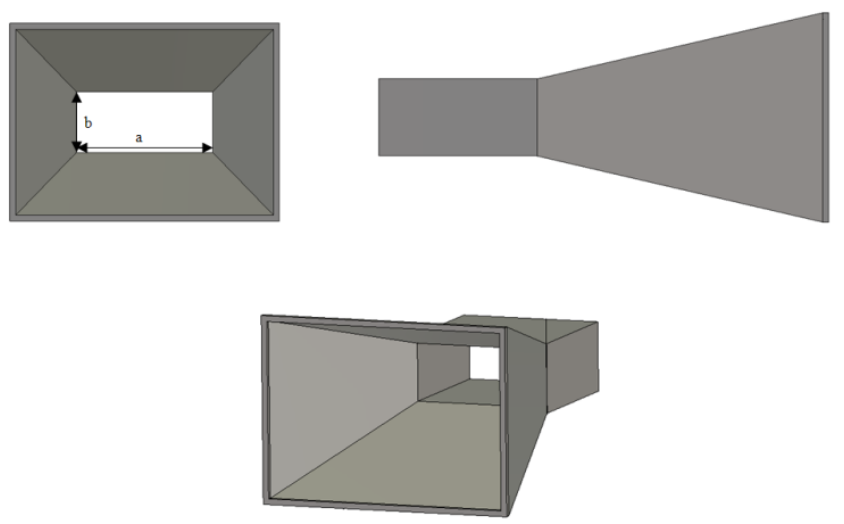

Figure 5 X-band pyramidal horn dimensions

Table 2 Horn antenna specifications

\begin{tabular}{cc}
\hline Specification & Horn Antenna \\
\hline Frequency, $\mathrm{GHz}$ & $8-12$ \\
Dimension, (a x b)mm & $22.86 \times 10.16$ \\
Radiation Pattern & Directive \\
\hline
\end{tabular}

A dual layer printed reflectarray consists of $35 \times 31$ printed minkowski (upper layer) and square (lower layer) microstrip patches on a dielectric substrate is shown in Figure 6. The chosen substrate was Taconic RF-35 with a thickness of $h=$ $1.524 \mathrm{~mm}$ and a dielectric constant $\varepsilon_{r}=3.54$. The employed technique for obtaining the progressive phase at each element was that of varying the element dimensions. The reflectarray was designed at $11 \mathrm{GHz}$ with the size of $400.05 \mathrm{~mm}$ x $297 \mathrm{~mm}$. The circular pattern built by the patches accounts for the compensation of the path differences in the almost spherical wave radiated backwards by the feed.

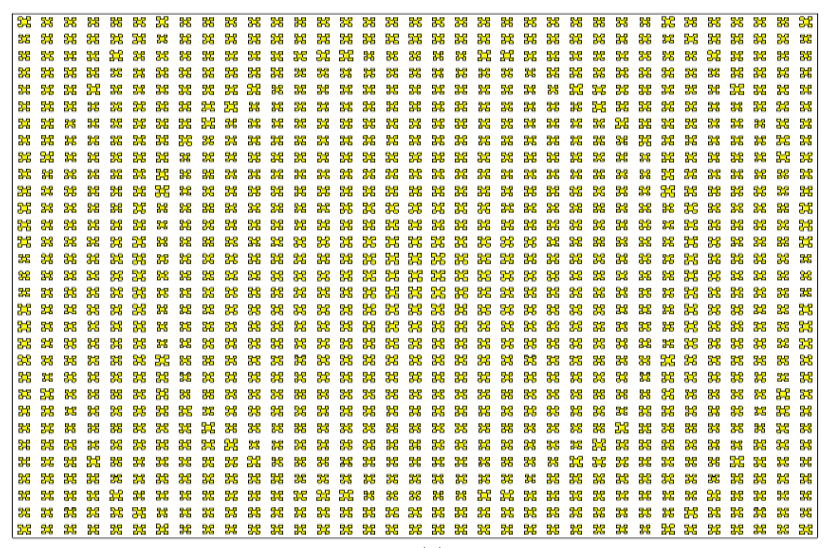

(a)

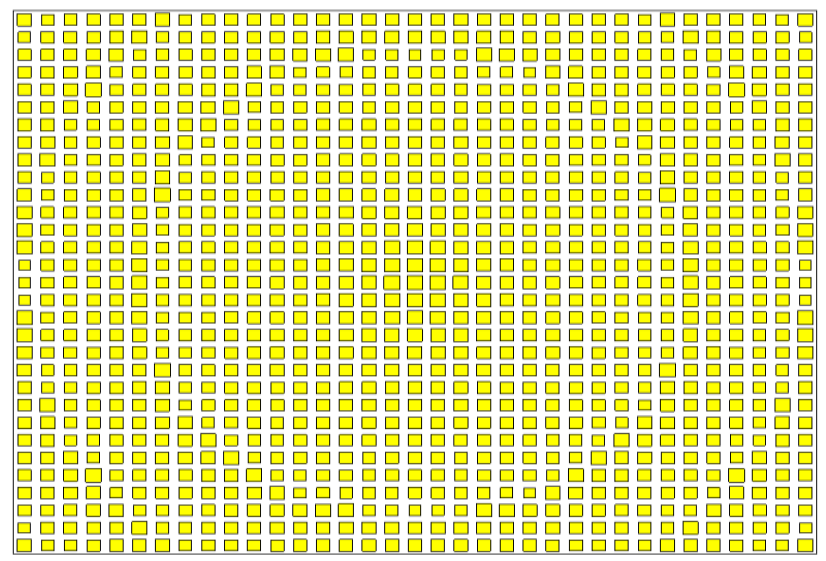

(b)

Figure 6 Reflectarray layouts. (a) Upper layer. (b) Lower layer with grounded plane

The reflectarray is designed so that the main beam points toward centre with fld ratio, 0.8. Simulation is performed in CST Mircowave Studio. From the simulation, a maximum gain of 25 $\mathrm{dB}$ turned out to occur at $11 \mathrm{GHz}$, which is the desired frequency. Since the best matching of the feed is at $11 \mathrm{GHz}$, Figure 7 shows the radiation pattern of this reflectarray at this frequency. A highly directive beam direct towards the centre of the horn antenna which is placed in front of the reflectarray. As could be observed in Figure 8, the HPBW of the antenna is $4.7^{\circ}$ in both plane which shows the beam is very narrow as expected for a reflectarray. For E-plane and H-plane, the side-lobe levels remain under $-18 \mathrm{~dB}$. 

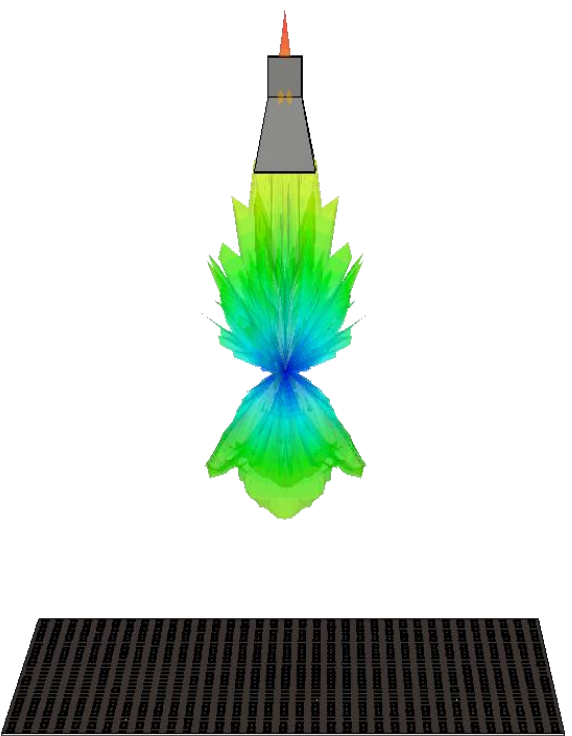

Figure 7 Radiation pattern in 3D

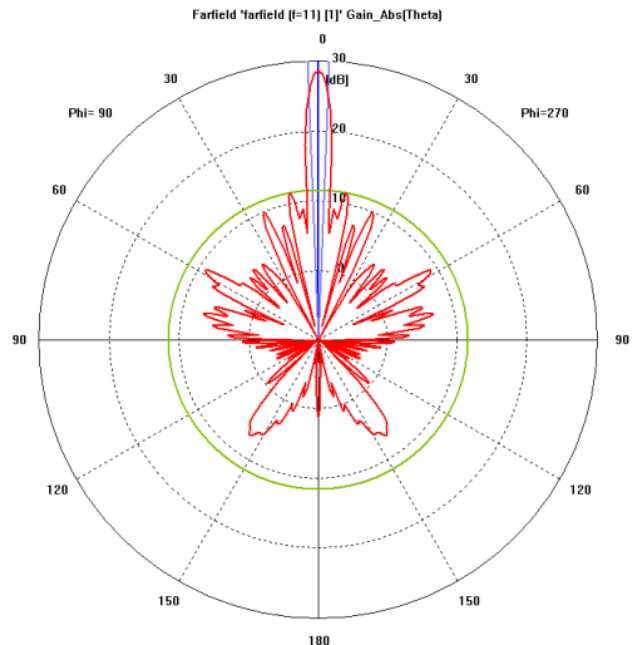

(a)

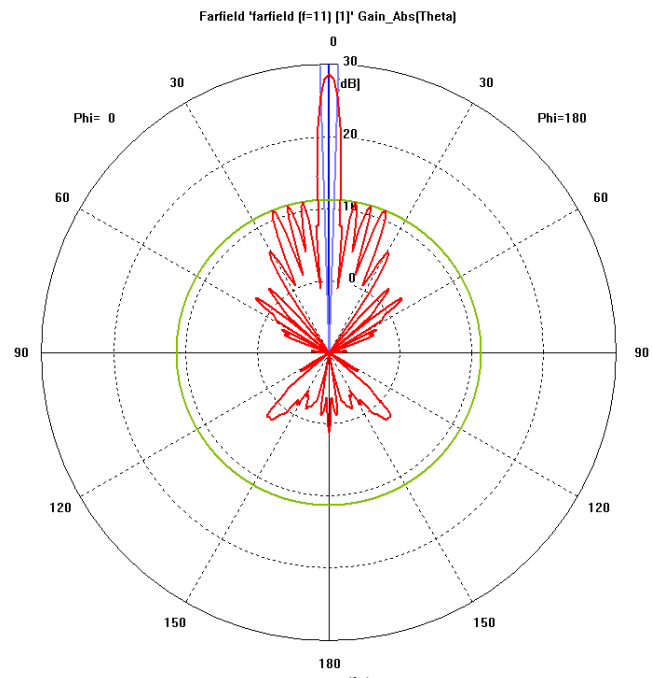

(b)

Figure 8 Polar plot. (a) E-plane. (b) H-plane
The side-lobes of the reflectarray are in both planes considerably high for a reflectarray, nevertheless it has acceptable values: $-17.2 \mathrm{~dB}$ in the Eplane and $-17.4 \mathrm{~dB}$ in the $\mathrm{H}-$ plane. Reasons for this high level of the side-lobes could be cause by the couplings between the microstrip radiating elements, which have not been taken into account in the design, or due to inaccuracy in the choice of the center feed. Its main disadvantage is that the displacements of the feed from the symmetry axis of the reflector maximize significantly the aperture blockage, thus reduced the efficiency. A summary of the properties of the simulated fixed-beam dual layer printed reflectarray with centre feed is presented in Table 3.

Table 3 Summary of the simulated dual layer printed reflectarray

\begin{tabular}{cc}
\hline Antenna & \multicolumn{1}{c}{$\begin{array}{c}\text { Dual Layer Printed } \\
\text { Reflectarray Antenna }\end{array}$} \\
\hline Substrate Type & \multicolumn{1}{c}{ Taconic RF-35 $\varepsilon_{r}=3.54$ tan $\delta=$} \\
Substrate Thickness & 0.0018 \\
SLL in E-Plane & $1.524 \mathrm{~mm}$ \\
SLL in H-Plane & $-17.2 \mathrm{~dB}$ \\
Directivity & $-17.4 \mathrm{~dB}$ \\
Gain & $26.1 \mathrm{dBi}$ \\
\hline
\end{tabular}

\subsection{CONCLUSION}

In this paper, two proposed dual layer printed unit cell configuration have been modeled in CST Mircowave Studio and the reflection coefficient results had been compared. An Infinite Array approach has been used to obtain reflection coefficient phase curves for a dual layer printed microstrip reflectarray with variable size patches. Based on this approach it is found out that when a Minkowski radiating element shape is introduced in the dual layer configuration a wider reflection phase more than $360^{\circ}$ is achieve. Following the reflection phase a two staked arrays composed of Minkowski and square patch, an centre feed dual layer printed microstrip reflectarray antenna with patches of variable size has been designed and simulated for operation at $11 \mathrm{GHz}$. This antenna featuring a highly directive centre beam radiation pattern and has shown good simulation performance. The achieved results for the unit cell and the reflectarray prove that Minkowski shape radiating element is a good candidate for enhancing reflection phase range in order to have a practical reflectarray design.

\section{Acknowledgements}

The authors thank to the Ministry of Higher Education Malaysia (MOHE), Ministry of Sceince, Technology and Innovation Malaysia (MOSTI), Research Management Centre (RMC) and Radio Communication Engineering Department, Universiti Teknologi Malaysia (UTM) for the supporting the research works under grant QJ13000.7123.02H02.

\section{References}

[1] D. M. Pozar and T. A. Metzler, 1993. Analysis of a reflectarray antenna using microstrip patches of variable size. Electron. Lett. 29(8): 657-658.

[2] D. M. Pozar, S. D. Targonski, and H. D. Syrigos, 1997. Design of millimeter wave microstrip reflectarrays. Proc. IEEE Trans. Antennas Propagat. 45: 287-295. 
[3] J. Huang, 1991 Microstrip reflectarray. In Proc. IEEE Int. Symp. Antennas Propagat. ON, Canada. 612-615.

[4] R. D. Javor, X. D. Wu, and K. Chang, 1995. Design and performance of a microstrip reflectarray antenna. IEEE Trans. Antennas Propagat. 43: 932-938.

[5] J. Huang, 1995. Bandwidth study of microstrip reflectarray and a novel phased reflectarray concept. In Proc. IEEE Int. Symp. Antennas Propagat. Newport Beach, CA. 582-585.
[6] F. Gautier et al. 1992. Phased reflector array and an antenna including such an array.US patent 5148 182, Sept.

[7] F. Zubir, M. K. A. Rahim, O. B. Ayop, H. A. Majid and A. Wahid. 2010. Design and analysis of microstrip refectarray antenna with minkowski shape radiation element. Progress In Electromagnetics Research B. 24: 317-331.

[8] J. A. Encinar, 1996. Design of a dual frequency reflectarray using microstrip stacked patches of variable size. Electron. Lett. 32: 10491050. 